\title{
Lockeia gigantus ichnosp. nov. in the Lacustrine Deposits of the Early Cretaceous Jinju Formation, Southern Coast of Korea
}

\author{
Kyung Soo Kim ${ }^{1}$ and Jeong Yul Kim ${ }^{2, *}$ \\ 'Chungbuk Science High School, Chungbuk 363-853, Korea \\ ${ }^{2}$ Department of Earth Science Education, Korea National University of Education, \\ Chungbuk 363-791, Korea
}

\section{남해안 전기 백악기 진주층의 호성 퇴적층에서 산출된 Lockeia gigantus ichnosp. nov.}

\author{
김경수 ${ }^{l} \cdot$ 김정률 $^{2, *}$ \\ 1충북과학고등학교, 363-853 충북 청원군 가덕면 상야리 \\ 2한국교원대학교 지구과학교육과, 363-371 충북 청원군 강내면 다락리
}

\begin{abstract}
About 450 specimens of Lockeia were discovered from the lacustrine siltstone of the Early Cretaceous Jinju Formation of Jin Island, southern coast of Korea. They are very elongated, seed-shaped Lockeia characterized by a large size, mostly $60-70 \mathrm{~mm}$ long and $15-20 \mathrm{~mm}$ wide. They are characteristically sharp longitudinal furrow bounded by steeply inclined both margins, elevated marginal rims and sharp pointed both longitudinal furrow ends. This trace fossil is herein described as Lockeia gigantus ichnosp. nov. Plicatounio, a freshwater bivalve which does not occur occurs occasionally within Lockeia gigantus is regarded as the most-likely producer of this resting trace fossil. This new trace fossil represents the largest Lockeia ever known and the first record of Lockeia from the Cretaceous non-marine deposits in the world. This fossil also represents an unusual example of resting trace fossil (Lockeia) associated with a possible producer (bivalve Plicatounio) lived in community in the shallow lacustrine environment.
\end{abstract}

Keywords: Lockeia gigantus ichnosp. nov., Plicatounio, non-marine, Jinju Formation, Cretaceous, Korea

요 약: 남해안의 경남 진도에 분포하는 전기 백악기 진주층의 호성 실트암에서 약 450여 개의 생흔 화석 Lockeia가 발 견되었다. 이 생흔 화석들은 상당히 길쪽한 씨앗 모양이며, 대부분 길이가 $60-70 \mathrm{~mm}$, 폭이 $15-20 \mathrm{~mm}$ 이다. 그들은 특징 적으로 날카로운 세로 고랑이 양쪽 가장자리에서 급하게 경사진 부분과 접하며, 주변부는 융기되어 있고, 세로 고랑의 양쪽 끝부분은 뽀족하다. 이 생흔 화석은 신종인 Lockeia gigantus ichnosp. nov.로 기재한다. Lockeia gigantus화 함께 드물게 발건되는 담수 이매패류인 Plicatounio는 이 휴식 생흔 화석의 가장 유력한 생성자로 관단된다. 이 신종 생흔 화석은 현재까지 알려진 Lockeia 중에서 가장 크고 백악기 육성 퇴적충에서 세계적으로 처음 보고된 Lockeia이다. 이 화석은 또한 얕은 호수 환경의 생물 군집에서 살았던 생흔 화석 생성자 (이매패류인 Plicatounio)와 관련된 휴식 생흔 화석의 특이한 예이다.

주요어: Lockeia gigantus ichnosp. nov., Plicatounio, 비해성, 진주층, 백악기, 한국

\section{Introduction}

Lockeia James, 1879, the senior synonym of

*Corresponding author: kimjy@knue.ac.kr

Tel: $82-43-230-3720$

Fax: 82-43-232-7176
Pelecypodichmus Seilacher, 1953, has been known as an ichnogenus of small almond-shaped oblong bodies preserved in convex hyporelief, tapering to sharp and obtuse points at both ends (Häntzschel, 1975). Although Lockeia was originally interpreted as an alga (James, 1879), it is now considered to be a resting 
trace of burrowing pelecypods (e.g. Seilacher, 1953; Osgood, 1970; Häntzschel, 1975; Vossler and Pemberton, 1988). Lockeia has received considerable attention in interpreting the life history, ecology, and ethology of bivalves (e.g. Eagar et al., 1983).

As shown in Table 1, there are numerous occurrences of Lockeia from fluvial to deep marine deposits from the Precambrian to Cretaceous age in North America (USA and Canada), Europe (U.K., Germany, Poland, and Switzerland), and Asia (Saudi Arabia, Tibet, and Korea). However, few analyses on its mode of formation exist.

Eight ichnospecies of Lockeia, L. siliquaria James, 1879, L. amygdaloides Seilacher, 1953, L. ornatus Bandel, 1967, L. czarnockii Karazewski, 1974, L. elongatus Yang, 1984, L. avalonensis Fillion and Pickerill, 1990, L. cordata Rindsberg, 1994, and L. triangulichnus Kim, 1994, are recognized in the current literature. L. ornatus characterized by its surface ornamentation (Bandel, 1967) and $L$. czarnockii, characterized by its large size and dropshaped form (Pienkowski, 1985) have been reported only from their type localities. L. elongatus is smooth, characteristically thin and elongate (Yang, 1984). L. avalonensis is a spherical, sub-ovate, squat Lockeia with an evenly rounded surface (Fillion and Pickerill, 1990). L. triangulichmus is a triangular trace resembling a small pyramid (Kim, 1994). Lockeia siliquaria and L. amygdaloides are well known from Early Ordovician to Cretaceous strata (e.g. Seilacher, 1953; Pickerill, 1977; Bromley and Asgaard, 1979; Crimes et al., 1981; Pienkowski, 1985; Fillion and Pickerill, 1990; Kim, 1994). L. cordata is a heart-shaped to a arrowhead-shaped Lockeia with nearly smooth surface (Rindsberg, 1994).

Most Lockeia populations consist of clusters of individual traces belonging to the same ichnospecies (e.g. James, 1879; Seilacher, 1953; Osgood, 1970). A unique population including a new trace fossil with numerous and diverse forms of Lockeia including $L$. siliquaria, L. amygdaloides, and L. triangulichnus was reported in Yeongweol, Korea (Kim, 1994).

The purpose of this paper is to describe a new species of Lockeia, L. gigantus ichnosp. nov. unusually associated with bivalves from the Cretaceous non-marine deposits of Korea. Like a very common situation in ichnology, there has been considerable debates on the producing animal of Lockeia. Although bivalves have been generally regarded as probable producers of some forms of Lockeia, bivalves associated with Lockeia have rarely been reported.

\section{Geologic Setting}

The Cretaceous Gyeongsang Supergroup occurs within the Gyeongsang Basin and several small basins (Haenam, Neungiu, Jinan, Kyokpo, Yongdong, Kongju, and Eumsung). The Gyeongsang Basin, the largest sedimentary basin of Korea, is located in the southeastern part of the Korean Peninsula.

The Gyeongsang Supergroup is divided into the Sindong, Hayang, and Yucheon groups. The first two groups consist mainly of thick siliciclastic sequences of alluvial, fluvial and lacustrine sediments, and the Yucheon Group characterized by the dominance of volcanic rocks (Chang, 1975; Fig. 1). The Sindong Group is confined to the western margin of the Gyeongsang Basin with general trends to the NNE (Nagdong Trough, 2,000 to 3,000 $\mathrm{m}$ thick). The Sindong Group consists of the Nagdong, Hasandong, and Jinju formations, generally showing a finingupward trend and three facies associations characterized as alluvial fan fringe, fluvial system, and shallow lake (Choi, 1986a, 1986b, 1986c). The source areas of the Sindong sediments have been revealed to WNW of the basin by paleocurrent analysis (Chang and Kim, 1968; Cheong and Kim, 1996). The occurrence of calcisol and vertisol indicates that climates during the Sindong deposition were arid to semi-arid (Paik and Lee, 1994, 1998; Paik and Kim, 1995). Abundant plant fossils collected from the Nagdong Formation were correlated from the floras of the Cretaceous Tetori Group in Japan (Tateiwa, 1925, 1929). The Sindong molluscan faunas have been studied extensively and all are assigned to non-marine taxa 


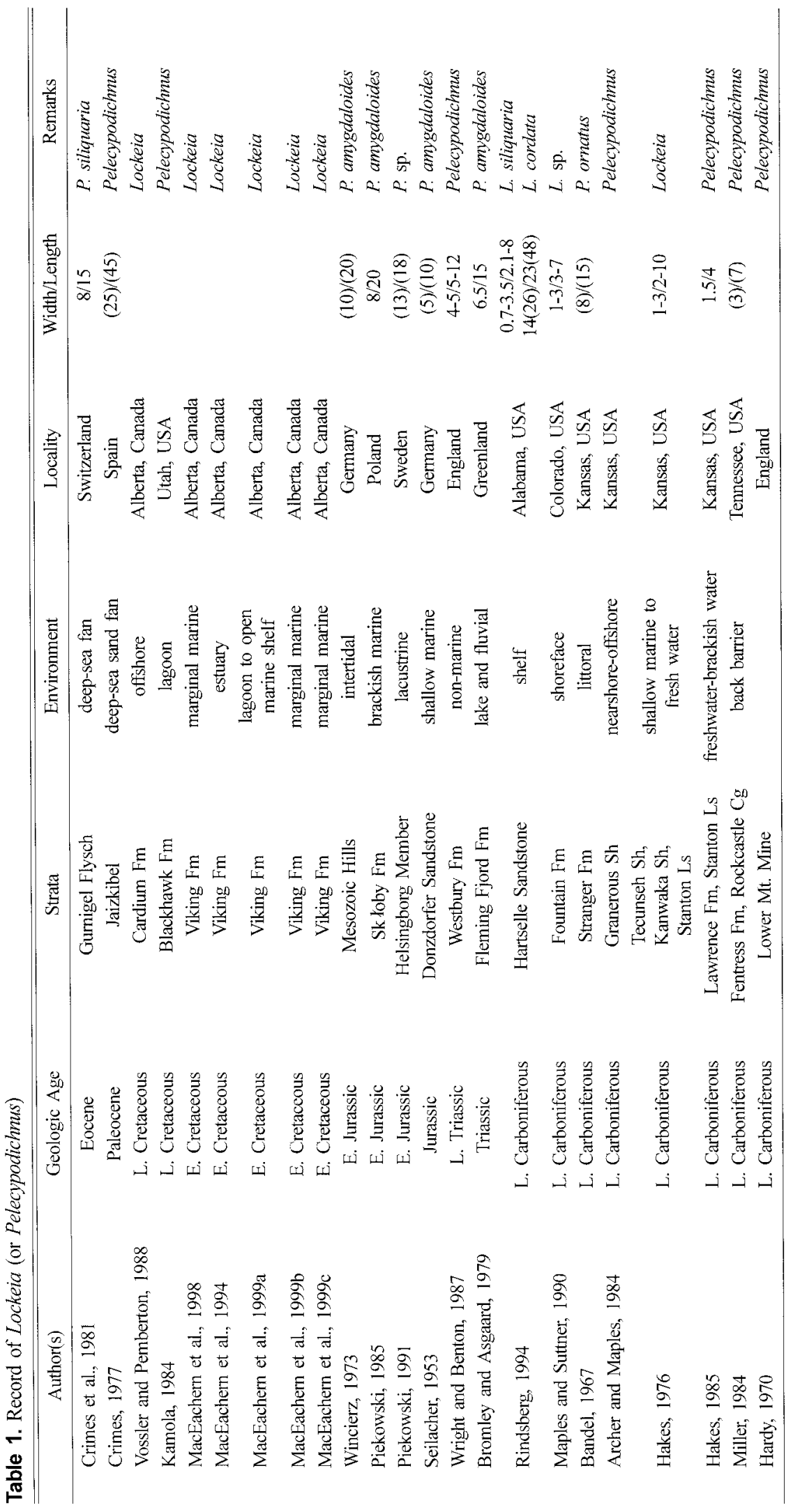


16 Kyung Soo Kim and Jeong Yul Kim

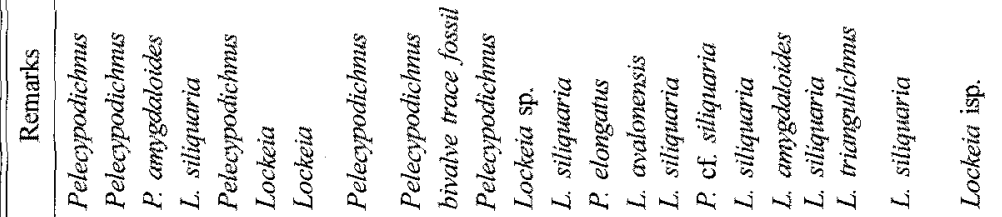

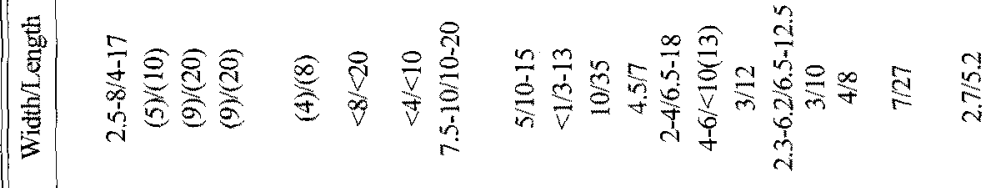

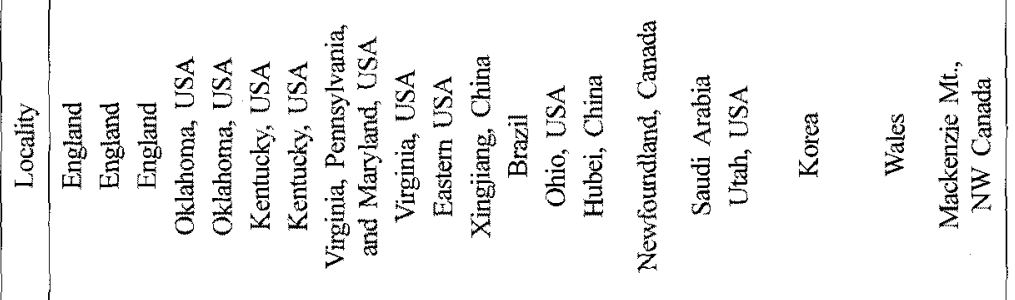

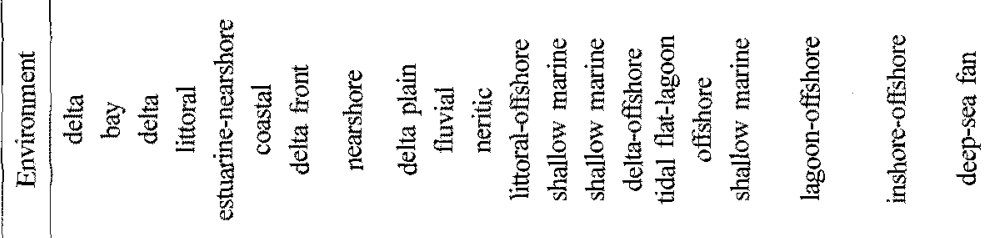

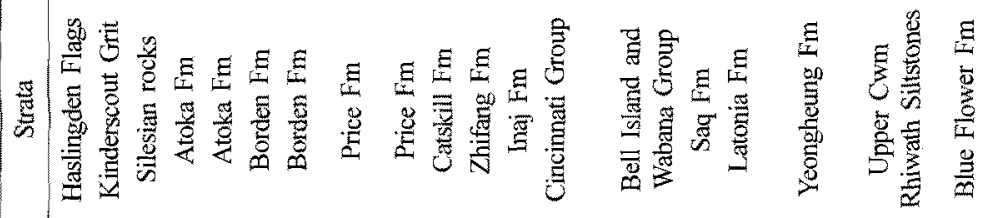

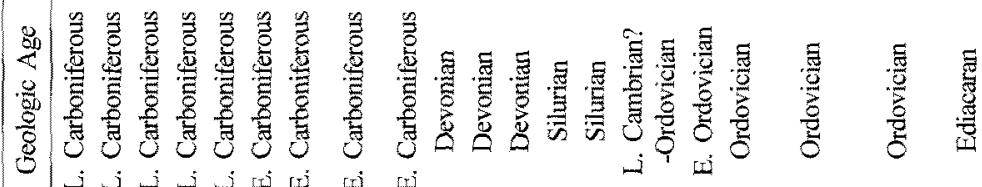

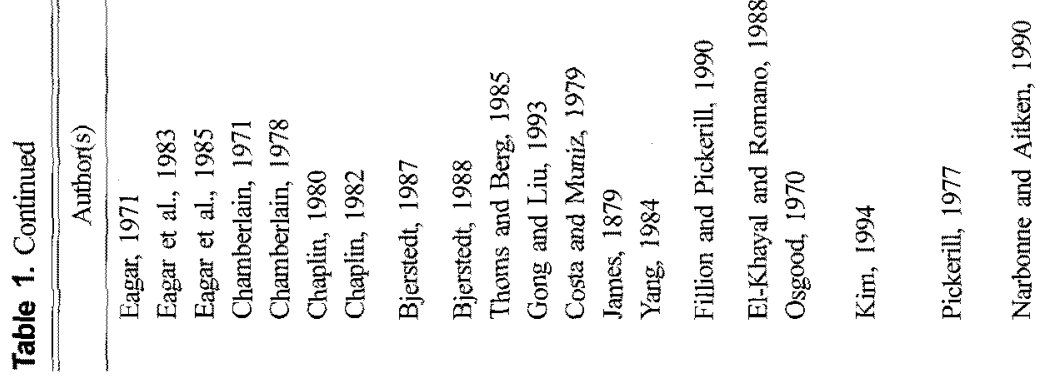




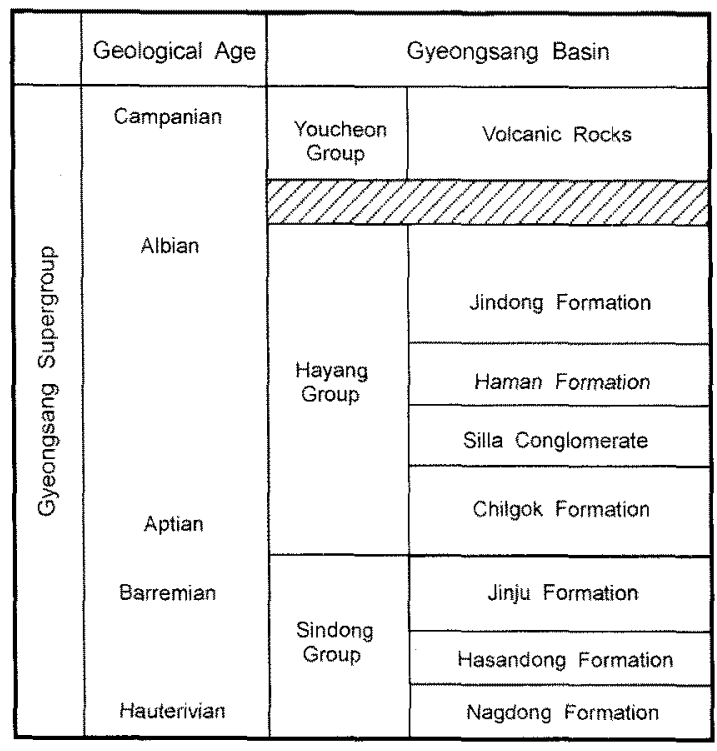

Fig. 1. Stratigraphy of the Gyeongsang Supergroup (after Chang, 1987).

(Yang, 1974, 1975, 1976, 1978a, 1978b, 1979, 1982). In addition, charophytes and non-marine ostracodes were also recovered from the Nagdong and Jinju formations, respectively (Choi, 1989, 1990). The age of the Sindong Group has been determined as Aptian to Albian by molluscan faunas (Yang, 1982) and as Hauterivian to Barremian by palynomorphs (Choi, 1985, 1989; Yi et al., 1994).

The Hayang Group overlies the Sindong Group in the western part of the Gyeongsang Basin and directly overlies pre-Cretaceous rocks in the northern part of the Gyeongsang Basin. This group (1,000 to $5,000 \mathrm{~m}$ in thickness) was deposited through subsiding and eastward expansion of the basin. It is composed of shale, sandstone interbedded with marlstone and conglomerate, and partly volcanic rocks extruded within the sedimentary basin. The paleocurrent directions indicate that dominant source areas were in the northwest and the east, somewhere in the vicinity of the present East Sea (Chang, 1988; Chang et al., 1990). During the sedimentation of the Hayang Group, the basin was strongly controlled by WNW-trending growth faults, which divided the basement into smaller crustal segments such as the Milyang, Euiseong, and
Yeongyang blocks. Therefore, under the influence of these syndepositional movements, the Hayang Group has different stratigraphic sequences from block to block (Chang, 1975, 1977).

The Hayang Group was deposited mainly in fluviolacustrine environments more than alluvial plains, an inference supported by abundant channel beds in association with floodplain sediments (Choi, 1986a). However, poorly sorted coarse grains of clastic rocks and prevalent reddish rock color suggest rather purely fluvial environments sometimes. The Hayang climate was generally arid to semi-arid judging from the occurrence of calcrete, rhizolith (Paik and Chun, 1993), and abundant pollen from Corollina and Ephedripites combined with scarcity of fern spores (Choi, 1985; Yi et al., 1993).

Since 40 specimens of plant fossils were reported from the Geoncheonri Formation and correlated with the Monobegawa Group and the Gyliak Series (Late Jurassic to Early Cretaceous) in Japan (Tateiwa, 1925, 1929), Hayang plant fossils have been recovered from the Geoncheonri, Daegu, and Iljik formations (Choi, 1989, 1990). Angiosperm pollens such as Retimonocolpites, Clavatipollenties, and Tricolpites from the Geoncheonri and Iljik formations suggest that the Hayang Group is Aptian to early Albian in age (Choi, 1985, 1989; Yi et al, 1994).

The Yucheon Group (2,000 to $3,000 \mathrm{~m}$ thick), unconformably overlying the Hayang Group, consists of volcanic and associated sedimentary rocks. No fossils are known so far from the Yucheon Group and its ${ }^{40} \mathrm{~K} /{ }^{40} \mathrm{Ar}$ radiometric age is $83 \mathrm{Ma}$ (Doh and Kim, 1994).

The Jin Island, the fossil locality of Lockeia, is located in the Hallyo Haesang National Park of the southern coast of the Korean Peninsula. The fossil site is located about $5 \mathrm{~km}$ west from the dinosaur track site (Lockley et al., 2006), world-famous Deogmyeongri of Sacheon City. The Jin Island is small, up to about $500 \mathrm{~m}$ in north-south length, and located in Bitori, Seopo-myeon, Sacheon City (Fig. 2). The fossil locality is placed at $128^{\circ} 58^{\circ} 26^{\prime \prime} \mathrm{E}, \mathrm{N} 34^{\circ} 57^{\prime} 34^{\prime \prime}$ in the southwest of the island. 


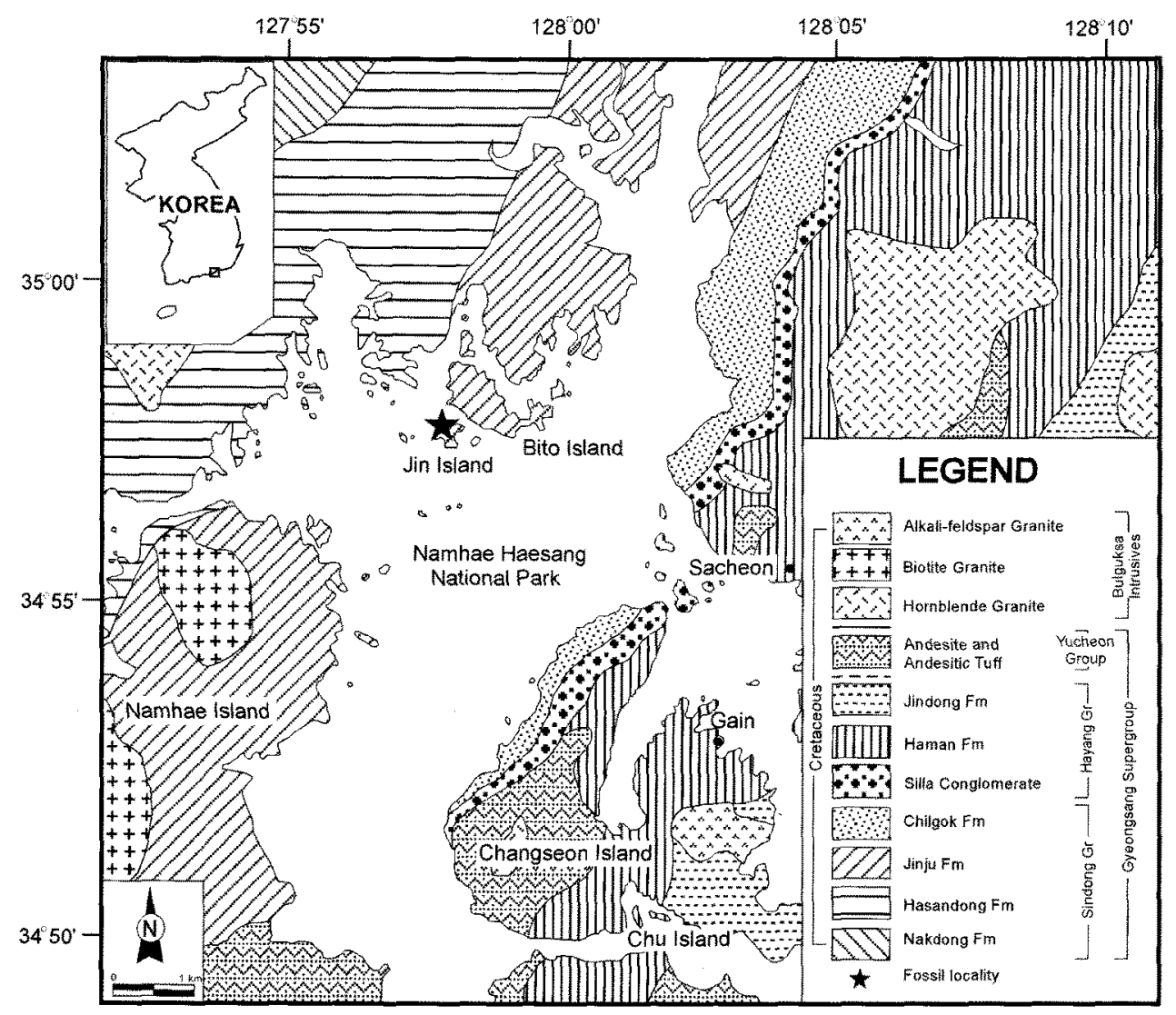

Fig. 2. Geologic map showing fossil locality (asterisk) (Kim et al., 1998 and Choi et al., 2002).

In the Jin Island, non-marine mollusks such as Trigonioides, Plicatounio, and Viviparus, theropod and sauropod tracks, and diverse types of stromatolites were observed from the Jinju Formation. In the fossil locality of the Jin Island, the lower part of the Jinju Formation predominately composed of mudstone infrequently intercalated with thin fine-grained sandstone. Strike and dip of fossil-bearing sandstone bed, probably deposited in the lacustrine margin environment, are $\mathrm{N} 30^{\circ} \mathrm{E}$ and $20^{\circ} \mathrm{SE}$.

\section{Occurrence of Lockeia}

Over 450 specimens of Lockeia are observed on the bedding surface in an area with about $500 \mathrm{~cm}$ in maximum length and $242 \mathrm{~cm}$ in maximum width (Fig. 3). Thus, fossil-bearing bed is about $6 \mathrm{~m}^{2}$ in area and density of Lockeia is about $82 / \mathrm{m}^{2}$. Assuming that the length of Lockeia gigantus is approximately five times those of currently well-known Lockeia (Fig. 4), it appears that the density of Lockeia gigantus specimens is regarded to be very high. It appears highly probable that these trace fossils were produced by communities of bivalves.

All specimens of Lockeia were measured and plotted in length-width diagram (Table 2, Fig. 3). The length of Lockeia is highly variable, ranges between $18 \mathrm{~mm}$ and $100 \mathrm{~mm}$ and about $50 \mathrm{~mm}$ in average. The width changes from $4 \mathrm{~mm}$ to $33 \mathrm{~mm}$ and about 13 $\mathrm{mm}$ in average. As shown is Fig. 4, it would appear that these are different size classes, perhaps representing cohorts of different ages, as the similar cases to the Pelecypodichmus from the uppermost Triassic rocks of Staffordshire of England (Wright and Benton, 1987). There may be eight size classes represented here, with modes correspond to $18-28,29-34,35-44,45-57,58-$ 
Table 2. Measurement of length $(\mathrm{L})$, width $(\mathrm{W})$, and orientation $(O)$ of Lockeia specimens from the Jinju Formation

\begin{tabular}{|c|c|c|c|c|c|c|c|c|c|c|c|c|c|c|c|}
\hline No & $\mathrm{L}(\mathrm{mm})$ & $\mathrm{W}(\mathrm{mm})$ & $\left.O 0^{\circ}\right)$ & No & $\mathrm{L}(\mathrm{mm})$ & $\mathrm{W}(\mathrm{mm})$ & 00 & No & $\mathrm{L}(\mathrm{mm})$ & $\mathrm{W}(\mathrm{mm})$ & 00 & No & $\mathrm{L}(\mathrm{mm})$ & $\mathrm{W}(\mathrm{mm})$ & 00 \\
\hline 1 & 49 & 13 & 85 & 56 & 71 & 10 & -30 & 111 & 42 & 15 & 19 & 166 & 41 & 11 & 7 \\
\hline 2 & 58 & 20 & 85 & 57 & 39 & 15 & 70 & 112 & 61 & 9 & 16 & 167 & 32 & 9 & -7 \\
\hline 3 & 65 & 15 & -70 & 58 & 60 & 12 & -40 & 113 & 53 & 8 & -69 & 168 & 61 & 12 & -26 \\
\hline 4 & 51 & 13 & 80 & 59 & 50 & 9 & -35 & 114 & 59 & 11 & 18 & 169 & 52 & 8 & -59 \\
\hline 5 & 45 & 10 & -80 & 60 & 45 & 15 & 27 & 115 & 45 & 13 & 43 & 170 & 62 & 14 & 11 \\
\hline 6 & 48 & 9 & 53 & 61 & 50 & 11 & -35 & 116 & 54 & 12 & 16 & 171 & 21 & 11 & 64 \\
\hline 7 & 45 & 11 & 68 & 62 & 40 & 11 & 80 & 117 & 55 & 19 & 55 & 172 & 41 & 13 & 78 \\
\hline 8 & 50 & 15 & -28 & 63 & 40 & 10 & 60 & 118 & 44 & 11 & 53 & 173 & 52 & 21 & -60 \\
\hline 9 & 59 & 15 & 4 & 64 & 39 & 12 & 20 & 119 & 52 & 10 & 21 & 174 & 32 & 12 & 23 \\
\hline 10 & 45 & 12 & 21 & 65 & 55 & 9 & 90 & 120 & 43 & 15 & 65 & 175 & 43 & 11 & 79 \\
\hline 11 & 40) & 11 & 76 & 66 & 54 & 15 & 12 & 121 & 51 & 12 & 40 & 176 & 43 & 18 & 73 \\
\hline 12 & 52 & 15 & 54 & 67 & 50 & 12 & -10 & 122 & 42 & 9 & 81 & 177 & 42 & il & 24 \\
\hline 13 & 40 & 12 & 70 & 68 & 22 & 8 & 50 & 123 & 33 & 11 & 29 & 178 & 24 & 7 & 43 \\
\hline 14 & 52 & 5 & -15 & 69 & 35 & 10 & -57 & 124 & 41 & 12 & 79 & 179 & 41 & 9 & -68 \\
\hline 15 & 68 & 12 & -35 & 70 & 43 & 9 & 15 & 125 & 43 & 8 & 31 & 180 & 42 & 10 & 28 \\
\hline 16 & 47 & 13 & 56 & 71 & 47 & 10 & -30 & 126 & 59 & 5 & -53 & 181 & 42 & 15 & -36 \\
\hline 17 & 23 & 8 & -75 & 72 & 38 & 9 & 44 & 127 & 32 & 11 & .80 & 182 & 51 & 12 & 4 \\
\hline 18 & 48 & 13 & 75 & 73 & 45 & 9 & -70 & 128 & 31 & 6 & -23 & 183 & 41 & 22 & 29 \\
\hline 19 & 45 & 15 & -71 & 74 & 20 & 5 & 25 & 129 & 42 & 8 & 70 & 184 & 32 & 12 & 27 \\
\hline 20 & 30 & 8 & -65 & 75 & 18 & 5 & -45 & 130 & 39 & 11 & -89 & 185 & 41 & 11 & 90 \\
\hline 21 & 63 & 20 & 30 & 76 & 41 & 8 & 90 & 131 & 51 & 9 & 16 & 186 & 32 & 5 & -73 \\
\hline 22 & 42 & 12 & 15 & 77 & 38 & 8 & 0 & 132 & 32 & 12 & 56 & 187 & 31 & 11 & 38 \\
\hline 23 & 22 & 5 & -80 & 78 & 60 & 8 & -25 & 133 & 62 & 11 & -31 & 188 & 34 & 10 & 44 \\
\hline 24 & 37 & 10 & 5 & 79 & 68 & 9 & -47 & 134 & 31 & 9 & 68 & 189 & 42 & 11 & 26 \\
\hline 25 & 40 & 9 & -75 & 80 & 49 & 12 & -67 & 135 & 42 & 11 & 29 & 190 & 24 & 16 & 73 \\
\hline 26 & 45 & 9 & -50 & 81 & 40 & 8 & -35 & 136 & 31 & 8 & -75 & 191 & 51 & 12 & 25 \\
\hline 27 & 73 & 10 & -60 & 82 & 40 & 12 & -70 & 137 & 41 & 7 & -38 & 192 & 61 & 11 & -19 \\
\hline 28 & 60 & 15 & 63 & 83 & 33 & 9 & 90 & 138 & 40 & 11 & -89 & 193 & 31 & 9 & -11 \\
\hline 29 & 35 & 8 & 24 & 84 & 28 & 7 & -88 & 139 & 41 & 10 & 36 & 194 & 42 & 11 & 18 \\
\hline 30 & 55 & 19 & -81 & 85 & 30 & 8 & -88 & 140 & 35 & 11 & 69 & 195 & 41 & 11 & -68 \\
\hline 31 & 27 & 7 & 15 & 86 & 47 & 8 & -53 & 141 & 41 & 9 & 4 & 196 & 53 & 10 & -73 \\
\hline 32 & 51 & 9 & -34 & 87 & 40 & 12 & 23 & 142 & 43 & 10 & 78 & 197 & 42 & 11 & -78 \\
\hline 33 & 49 & 9 & -84 & 88 & 47 & 10 & 0 & 143 & 51 & 11 & -17 & 198 & 21 & 8 & -86 \\
\hline 34 & 45 & 13 & 82 & 89 & 25 & 9 & 60 & 144 & 42 & 22 & 47 & 199 & 23 & 7 & -87 \\
\hline 35 & 31 & 8 & -75 & 90 & 25 & 8 & -10 & 1.45 & 61 & 15 & -14 & 200 & 71 & 8 & -45 \\
\hline 36 & 38 & 12 & -38 & 91 & 30 & 13 & 62 & 146 & 53 & 10 & 5 & 201 & 39 & 9 & -34 \\
\hline 37 & 40 & 10 & -15 & 92 & 40 & 10 & 35 & 147 & 61 & 15 & -19 & 202 & 31 & 8 & -90 \\
\hline 38 & 45 & 8 & 23 & 93 & 59 & 8 & 82 & 148 & 25 & 12 & 82 & 203 & 59 & 11 & -27 \\
\hline 39 & 38 & 7 & -75 & 94 & 38 & 10 & -8 & 149 & 82 & 13 & -39 & 204 & 29 & 9 & 0 \\
\hline 40 & 56 & 15 & 78 & 95 & 45 & 9 & -36 & 150 & 62 & 9 & -18 & 205 & 45 & 12 & -2 \\
\hline 41 & 49 & 10 & 90 & 96 & 40 & 9 & 54 & 151 & 31 & 11 & 9 & 206 & 24 & 11 & 64 \\
\hline 42 & 51 & 15 & 80 & 97 & 49 & 10 & -20 & 152 & 42 & 11 & -16 & 207 & 21 & 5 & -13 \\
\hline 43 & 48 & 10 & 57 & 98 & 48 & 10 & 23 & 153 & 41 & 7 & -40 & 208 & 34 & 10 & 29 \\
\hline 44 & 47 & 9 & -53 & 99 & 32 & 9 & 38 & 154 & 73 & 12 & -41 & 209 & 62 & 9 & 84 \\
\hline 45 & 45 & 12 & 50 & 100 & 50 & 9 & -65 & 155 & 32 & 11 & 21 & 210 & 33 & 10 & 52 \\
\hline 46 & 49 & 8 & 25 & 101 & 45 & 15 & 70 & 156 & 42 & 4 & -49 & 211 & 48 & 8 & -44 \\
\hline 47 & 28 & 8 & 56 & 102 & 42 & 9 & 15 & 157 & 52 & 11 & -6 & 212 & 38 & 9 & 54 \\
\hline 48 & 25 & 7 & 30 & 103 & 35 & 8 & -78 & 158 & 51 & 11 & -62 & 213 & 21 & 11 & -87 \\
\hline 49 & 38 & 10 & 40 & 104 & 35 & 10 & 43 & 159 & 41 & 12 & 10 & 214 & 53 & 18 & 82 \\
\hline 50 & 34 & 9 & 0 & 105 & 40 & 10 & 48 & 160 & 49 & 11 & 0 & 215 & 47 & 9 & -23 \\
\hline 51 & 48 & 8 & -20 & 106 & 31 & 9 & 72 & 161 & 38 & 11 & 51 & 216 & 31 & 11 & -3 \\
\hline 52 & 45 & 9 & 80 & 107 & 29 & 7 & -86 & 162 & 32 & 9 & -41 & 217 & 42 & 11 & 81 \\
\hline 53 & 50 & 18 & 90 & 108 & 38 & 8 & 80 & 163 & 21 & 4 & 37 & 218 & 35 & 12 & 32 \\
\hline 54 & 29 & 13 & .70 & 109 & 49 & 8 & -23 & 164 & 31 & 11 & 69 & 219 & 21 & 7 & 26 \\
\hline 55 & 60 & 25 & 55 & 110 & 41 & 9 & 71 & 165 & 39 & 8 & -22 & 220 & 24 & 9 & 62 \\
\hline
\end{tabular}


Table 2. Continued

\begin{tabular}{|c|c|c|c|c|c|c|c|c|c|c|c|c|c|c|c|}
\hline No & $\mathrm{L}(\mathrm{mm})$ & $\mathrm{W}(\mathrm{mm})$ & 00 & No & $\mathrm{L}(\mathrm{mm})$ & $\mathrm{W}(\mathrm{mm})$ & 00 & No & $\mathrm{L}(\mathrm{mm})$ & $\mathrm{W}(\mathrm{mm})$ & 00 & No & $L(\mathrm{~mm})$ & $\mathrm{W}(\mathrm{mn})$ & 00 \\
\hline 221 & 42 & 9 & 24 & 279 & 43 & 18 & 52 & 337 & 50 & 25 & 70 & 395 & 50 & 15 & 55 \\
\hline 222 & 41 & 11 & 71 & 280 & 47 & 8 & -2 & 338 & 45 & 10 & 80 & 396 & 80 & 15 & 15 \\
\hline 223 & 38 & 10 & -88 & 281 & 39 & 15 & -86 & 339 & 45 & 8 & 55 & 397 & 60 & 15 & -37 \\
\hline 224 & 41 & 11 & 15 & 282 & 42 & 15 & -88 & 340 & 70 & 20 & 45 & 398 & 50 & 10 & -10 \\
\hline 225 & 72 & 11 & 43 & 283 & 56 & 12 & 8 & 341 & 50 & 8 & 90 & 399 & 40 & 5 & -35 \\
\hline 226 & 70 & 25 & 9 & 284 & 62 & 15 & 43 & 342 & 60 & 10 & 40 & 400 & 60 & 10 & -77 \\
\hline 227 & 40 & 11 & -76 & 285 & 52 & 25 & 53 & 343 & 55 & 10 & -65 & 401 & 45 & 10 & 90 \\
\hline 228 & 45 & 12 & -74 & 286 & 30 & 9 & -70 & 344 & 75 & 20 & 38 & 402 & 45 & 15 & 90 \\
\hline 229 & 35 & 8 & -76 & 287 & 40 & 9 & 10 & 345 & 60 & 10 & 12 & 403 & 50 & 15 & -25 \\
\hline 230 & 40 & 9 & -78 & 288 & 30 & 8 & -66 & 346 & 100 & 20 & -10 & 404 & 50 & 15 & -52 \\
\hline 231 & 35 & 9 & 10 & 289 & 30 & 8 & 12 & 347 & 65 & 10 & 0 & 405 & 75 & 15 & -28 \\
\hline 232 & 38 & 10 & 11 & 290 & 30 & 8 & -72 & 348 & 60 & 10 & 0 & 406 & 50 & 20 & 54 \\
\hline 233 & 35 & 10 & 90 & 291 & 35 & 10 & 59 & 349 & 70 & 10 & 0 & 407 & 55 & 15 & -58 \\
\hline 234 & 67 & 10 & -47 & 292 & 30 & 7 & -27 & 350 & 55 & 15 & 82 & 408 & 60 & 20 & -72 \\
\hline 235 & 58 & 17 & 68 & 293 & 45 & 7 & -32 & 351 & 80 & 15 & 0 & 409 & 65 & 15 & -10 \\
\hline 236 & 50 & 10 & 14 & 294 & 42 & 10 & 12 & 352 & 70 & 20 & 22 & 410 & 50 & 20 & 74 \\
\hline 237 & 42 & 16 & 61 & 295 & 25 & 8 & 13 & 353 & 90 & 15 & 15 & 411 & 45 & 25 & 43 \\
\hline 238 & 35 & 9 & 68 & 296 & 40 & 15 & 33 & 354 & 65 & 10 & 5 & 412 & 65 & 20 & 0 \\
\hline 239 & 52 & 14 & -54 & 297 & 42 & 15 & 85 & 355 & 60 & 20 & 10 & 413 & 55 & 20 & -82 \\
\hline 240 & 66 & 10 & -55 & 298 & 49 & 8 & 4 & 356 & 45 & 15 & 8 & 414 & 40 & 15 & -72 \\
\hline 241 & 78 & 21 & -24 & 299 & 31 & 10 & 37 & 357 & 45 & 15 & 40 & 415 & 60 & 15 & -68 \\
\hline 242 & 51 & 15 & -90 & 300 & 40 & 8 & -44 & 358 & 70 & 25 & -80 & 416 & 65 & 10 & -36 \\
\hline 243 & 50 & 12 & 64 & 301 & 34 & 8 & -36 & 359 & 60 & 20 & 50 & 417 & 45 & 20 & 40 \\
\hline 244 & 43 & 12 & -82 & 302 & 35 & 11 & 66 & 360 & 50 & 20 & 70 & 418 & 55 & 10 & 0 \\
\hline 245 & 51 & 12 & -87 & 303 & 55 & 18 & 66 & 361 & 60 & 15 & 20 & 419 & 50 & 10 & 32 \\
\hline 246 & 45 & 15 & 60 & 304 & 63 & 15 & -27 & 362 & 70 & 20 & 90 & 420 & 40 & 20 & 28 \\
\hline 247 & 58 & 11 & 73 & 305 & 90 & 23 & 5 & 363 & 50 & 20 & 78 & 421 & 55 & 15 & 49 \\
\hline 248 & 62 & 14 & -13 & 306 & 45 & 9 & 29 & 364 & 45 & 15 & 62 & 422 & 50 & 15 & 0 \\
\hline 249 & 55 & 25 & 27 & 307 & 48 & 15 & -82 & 365 & 40 & 10 & -15 & 423 & 40 & 10 & 65 \\
\hline 250 & 38 & 9 & 7 & 308 & 49 & 12 & -74 & 366 & 60 & 25 & 38 & 424 & 40 & 5 & 40 \\
\hline 251 & 65 & 19 & -8 & 309 & 49 & 14 & -88 & 367 & 45 & 10 & 85 & 425 & 50 & 10 & 28 \\
\hline 252 & 45 & 15 & 19 & 310 & 31 & 15 & -90 & 368 & 45 & 15 & 68 & 426 & 60 & 15 & -62 \\
\hline 253 & 61 & 25 & 28 & 311 & 40 & 12 & 43 & 369 & 70 & 15 & 90 & 427 & 45 & 10 & -5 \\
\hline 254 & 58 & 17 & .56 & 312 & 49 & 15 & 90 & 370 & 40 & 20 & 58 & 428 & 50 & 15 & -30 \\
\hline 255 & 37 & 15 & 40 & 313 & 42 & 9 & 22 & 371 & 90 & 30 & -47 & 429 & 40 & 10 & 60 \\
\hline 256 & 40 & 18 & -62 & 314 & 50 & 12 & 90 & 372 & 70 & 20 & -5 & 430 & 40 & 10 & 70 \\
\hline 257 & 58 & 10 & -57 & 315 & 60 & 12 & -65 & 373 & 30 & 15 & 45 & 431 & 45 & 15 & 30 \\
\hline 258 & 50 & 15 & -85 & 316 & 49 & 10 & 90 & 374 & 60 & 15 & 27 & 432 & 45 & 20 & 62 \\
\hline 259 & 38 & 10 & -83 & 317 & 50 & 12 & 90 & 375 & 60 & 20 & 27 & 433 & 40 & 15 & 56 \\
\hline 260 & 45 & 9 & -66 & 318 & 40 & 12 & 68 & 376 & 60 & 20 & 48 & 434 & 55 & 25 & 55 \\
\hline 261 & 50 & 10 & 43 & 319 & 70 & 20 & 36 & 377 & 60 & 20 & -88 & 435 & 45 & 10 & 88 \\
\hline 262 & 77 & 16 & 43 & 320 & 50 & 20 & 46 & 378 & 65 & 26 & 0 & 436 & 45 & 20 & 42 \\
\hline 263 & 40 & 7 & 45 & 321 & 40 & 10 & 64 & 379 & 50 & 25 & 25 & 437 & 40 & 20 & 40 \\
\hline 264 & 38 & 12 & 81 & 322 & 50 & 15 & 75 & 380 & 65 & 20 & 47 & 438 & 40 & 10 & 40 \\
\hline 265 & 59 & 10 & -28 & 323 & 60 & 20 & 57 & 381 & 55 & 20 & 38 & 439 & 50 & 15 & 75 \\
\hline 266 & 65 & 11 & -35 & 324 & 50 & 10 & 90 & 382 & 60 & 15 & 12 & 440 & 45 & 15 & 90 \\
\hline 267 & 52 & 15 & -18 & 325 & 70 & 12 & 90 & 383 & 70 & 15 & -45 & 441 & 35 & 15 & 90 \\
\hline 268 & 37 & 9 & 75 & 326 & 50 & 10 & 90 & 384 & 50 & 20 & 55 & 442 & 30 & 10 & 62 \\
\hline 269 & 48 & 12 & -73 & 327 & 70 & 12 & 70 & 385 & 60 & 30 & 25 & 443 & 60 & 10 & 0 \\
\hline 270 & 35 & 8 & 38 & 328 & 70 & 20 & -65 & 386 & 75 & 25 & 0 & 444 & 40 & 10 & 50 \\
\hline 271 & 32 & 9 & 53 & 329 & 60 & 20 & 38 & 387 & 65 & 15 & -13 & 445 & 35 & 10 & 35 \\
\hline 272 & 42 & 10 & 84 & 330 & 70 & 25 & -72 & 388 & 65 & 15 & -28 & 446 & 50 & 15 & 75 \\
\hline 273 & 39 & 9 & 84 & 331 & 60 & 25 & 58 & 389 & 30 & 20 & 46 & 447 & 40 & 10 & 70 \\
\hline 274 & 41 & 15 & 27 & 332 & 70 & 30 & -85 & 390 & 60 & 15 & -8 & 448 & 45 & 10 & 12 \\
\hline 275 & 30 & 8 & 41 & 333 & 60 & 15 & 90 & 391 & 50 & 10 & 90 & 449 & 20 & 10 & 50 \\
\hline 276 & 51 & 16 & 75 & 334 & 60 & 15 & 20 & 392 & 50 & 20 & 62 & 450 & 35 & 10 & -60 \\
\hline 277 & 62 & 9 & -58 & 335 & 70 & 20 & 0 & 393 & 50 & 10 & 33 & & & & \\
\hline 278 & 48 & 8 & -52 & 336 & 80 & 10 & 20 & 394 & 40 & 5 & 90 & & & & \\
\hline
\end{tabular}

* Note: Zero degree in orientation represents north. $(-)$ represents west. 


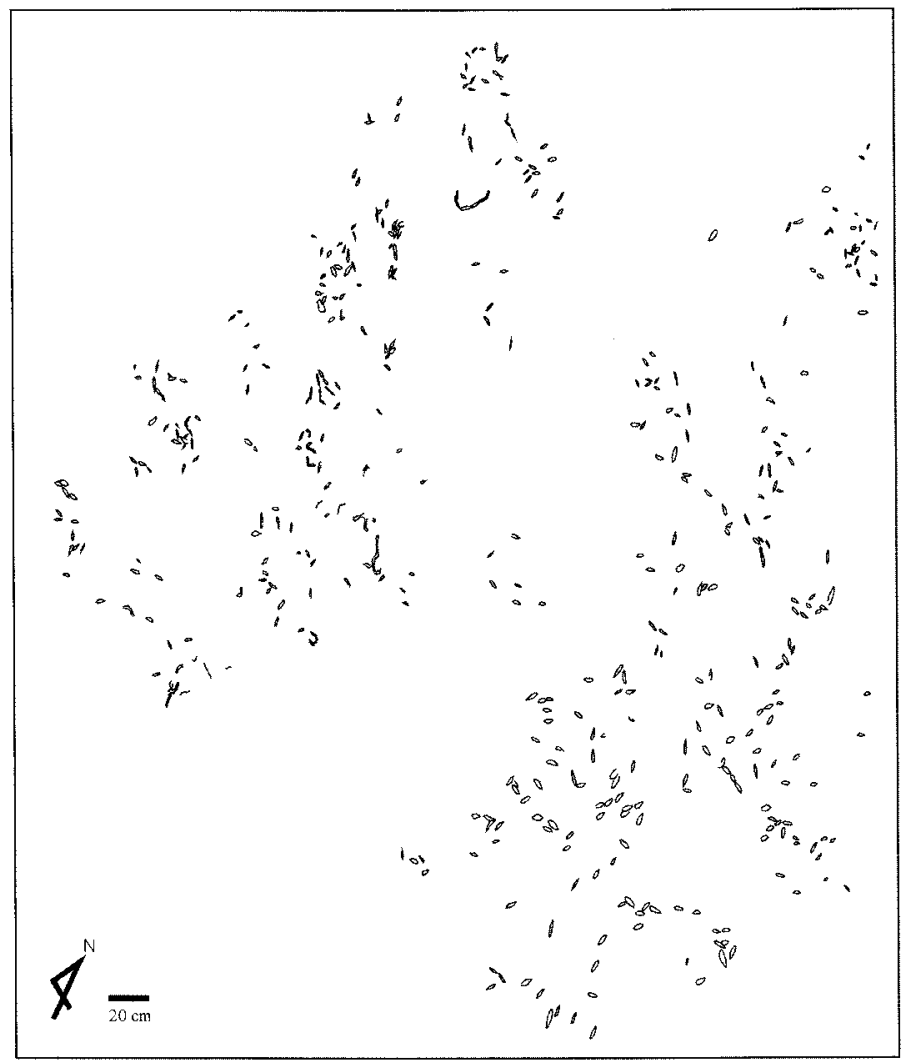

Fig. 3. Drawing of Lockeia on the bedding surface.

64, 65-74, 75-83, and 91-100 mm. Fig. 4 also shows the length/width of Lockeia previously reported by others showing the measured data for comparing Lockeia gigantus which is remarkably larger in size.

The specimens show preferred orientation faintly though it varies from place to place (Fig. 5). NNESWW and NEE-SWW trending orientations are most predominate, however, NW-SW orientation is also conspicuous. Orientations may be related to either a feeding and resting behavior or a more stable posture, like their analogues in modern and ancient streams and rivers (Pryor, 1967; Eagar et al., 1983). The relationship between preferred orientation and lake shoreline appears to be not ruled out, though sedimentary features indicating paleocurrent are not observed.

All of Lockeia specimens are preserved as concave epirelief on the fine-grained sandstone bed. Many of them are characterized by symmetrical, seed-like to almond-shaped forms commonly with a sharp longitudinal furrow and elevated marginal rims (Fig. 6), like plow-up ridges observed in modern environment (Pryor, 1967).

\section{Systematic Description}

Lockeia gigantus ichnosp. nov.

Fig. 6

Type material: Holotype (Fig. 6D) KNUE 060701, paratypes (Fig. 6B, E) KNUE 060702, 060703, same horizon and same locality as holotype. Silicon rubber mold are housed in the Paleontological Collections of Department of Earth Science Education, Korea National University of Education (KNUE).

Other material: about five hundreds specimens in the field at fossil locality. 


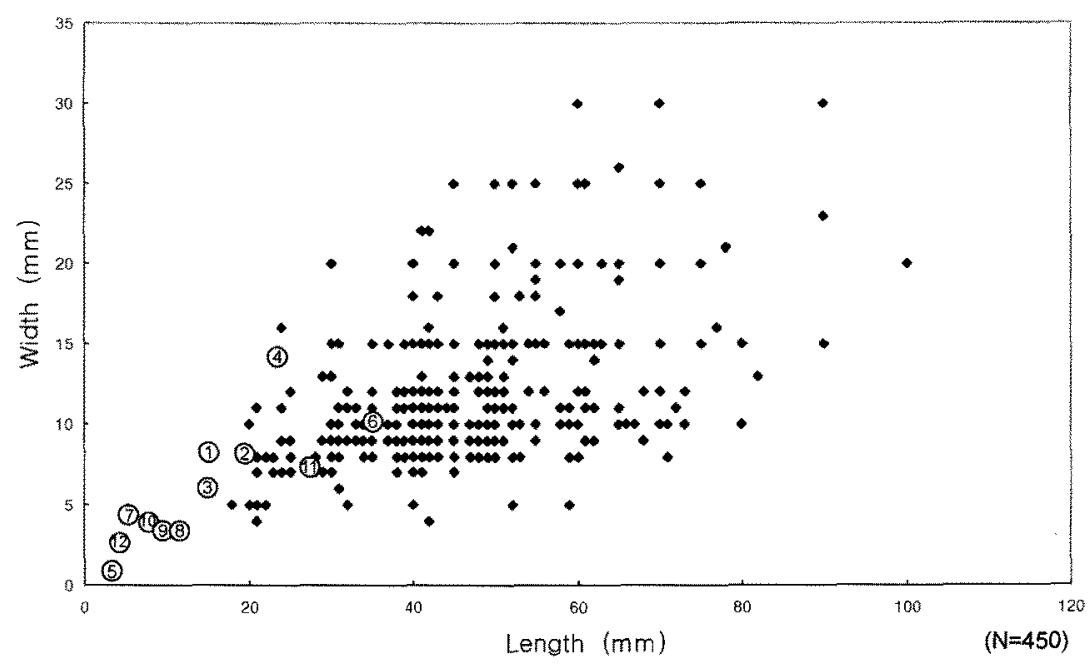

Fig. 4. Length/width diagram of Lockeia specimens $(\mathrm{N}=450)$ from the Jinju Formation and Lockeia or Pelecypodichmus previously reported by others (numbered as 1 to 12). 1: Pelecypodichmus siliquaria (Crimes et al., 1981), 2: P. amygdaloides (Pienkowski, 1985), 3: P. amygdaloides (Bromley and Asgaard, 1979) 4: L. cordata (Rindsberg, 1994), 5: P. (Hakes, 1985), 6: P. elongatis (Yang, 1984), 7: L. avalonensis (Fillion and Pickerill, 1990), 8: L. siliquaria (Osgood, 1970), 9: L. siliquaria (Kim, 1994), 10: L. tricmgulichnus (Kim, 1994), 11: L. siliquaria (Pickerill, 1977), 12: L. isp. (Narbonne and Aitken, 1990)

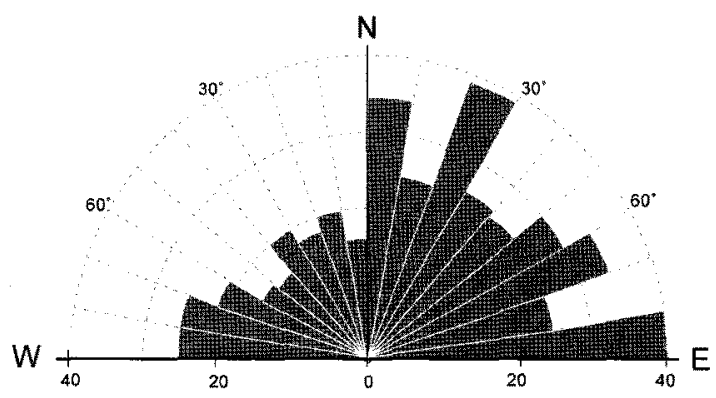

Fig. 5. Orientation of Lockeia specimens from the Jinju Formation. Numbers $(0,20$, and 40$)$ indicate number of specimens measured.

Derivation of name: Afler giant, large-sized Lockeia.

Occurrence: lower part of the Early Cretaceous Jinju Formation, Jin Island, Bitori, Seopo-myeon, Sacheon City, Korea.

Diagnosis: Large, seed-like to almond-shaped Lockeia with length up to $70 \mathrm{~mm}$ and width up to 30 $\mathrm{mm}$, preserved as concave epirelief. Longitudinal furrows or grooves commonly developed in the center along the midline of oblong body, which is V- to Ushaped in cross section and sided by elevated marginal rims commonly developed in both sides.

Description: Specimens of Lockeia gigantus ichnosp. nov. are preserved as concave epirelief on the $15 \mathrm{~cm}$ thick fine-grained sandstone intercalated within thick mudstone. They are typically symmetrical seedlike to almond-shaped, tapering at both ends to a sharp point. Well preserved specimens possess a sharp longitudinal furrow or groove (about $1.5 \mathrm{~mm}$ thick) along the elongate body which is $\mathrm{V}$ - to U-shaped in transverse section. Many specimens show marginal rims (about $10 \mathrm{~mm}$ wide and $5 \mathrm{~mm}$ high) elevated at both sides of oblong body. Length ranges between 30 to $70 \mathrm{~mm}$ (mostly 40 to $50 \mathrm{~mm}$ ), width varies from 10 to $30 \mathrm{~mm}$ (mostly 10 to $2 \mathrm{~mm}$ ), and depth is up to about $7 \mathrm{~mm}$.

Remarks: Since Lockeia James, 1879, the senior synonym of Pelecypodichmus, introduced as an ichnogenus of small almond-shaped oblong bodies tapering to sharp and obtuse points at both ends (Hntzschel, 1975). Although eight ichnospecies of Lockeia were erected, neither type material nor diagnosis and measured data were provided for 

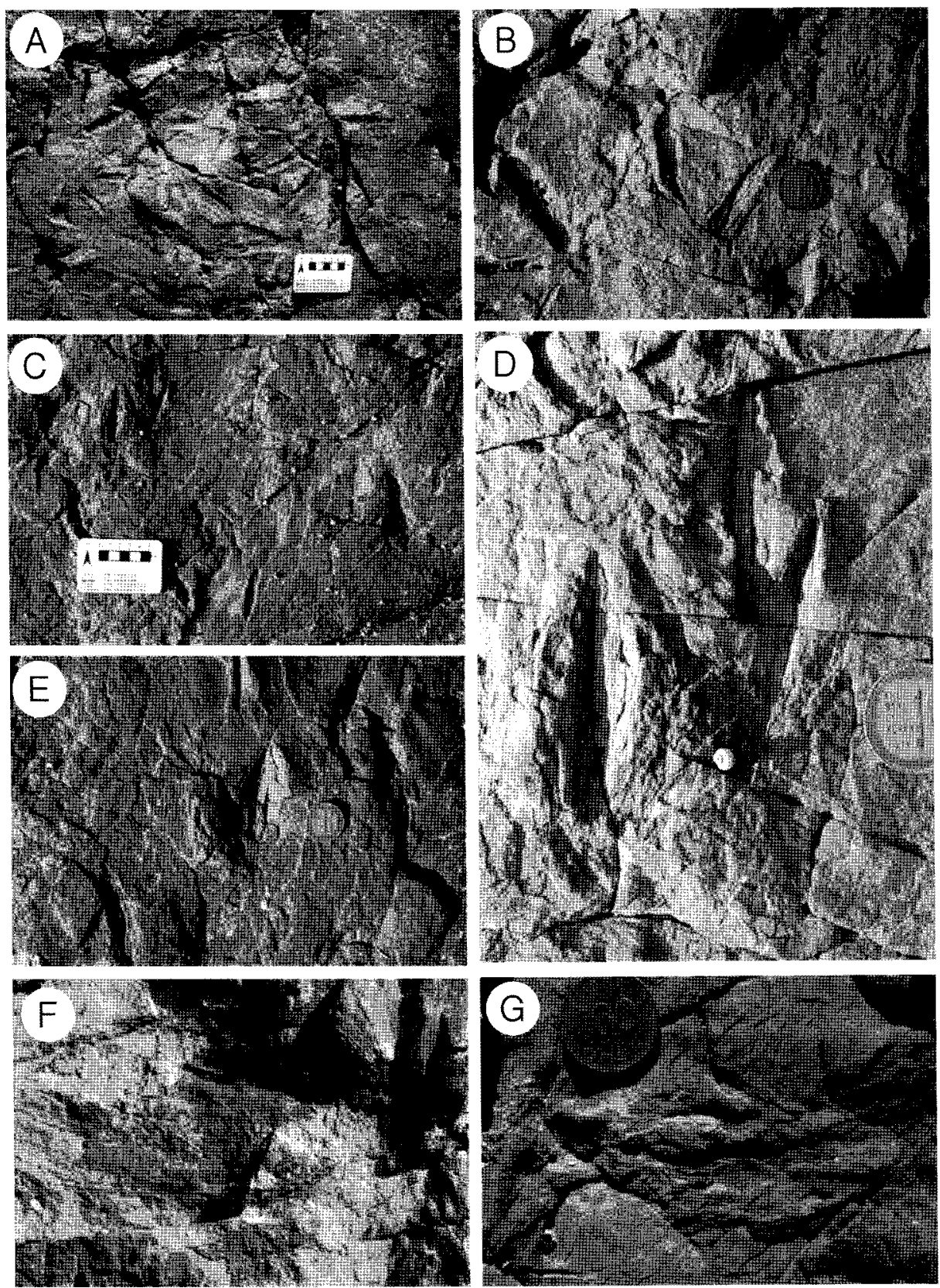

Fig. 6. Outcrop view of Lockeia gigantus (A E) and a possible trace maker, Plicatounio sp. preserved in situ $(\mathrm{F} \sim \mathrm{G})$.

systematic study on new ichnospecies of Lockeia. Whether described features of Lockeia czarnockii (Pienkowski, 1985) do or do not fall into diagnostic features of Lockeia is also regarded to be questionable in its validity for a new ichnospecies. Lockeia ornatus (Bandel, 1967) described from the Pennsylvanian sandstones of Kansas is a questionable ribbed from that may, in fact, belong to Walcottia Miller and Dyer 1878 (Fillion and Pickerill, 1990).

Specimens herein assigned to Lockeia gigantus have essential features corresponding to diagnosis of ichnogenus Lockeia. However, its comparison with currently known ichnospecies is not easy because of its characteristic morphology clearly distinguished 
from them. As the name of ichnospecies shows, Lockeia gigantus is the largest, gigantic Lockeia with length up to $70 \mathrm{~mm}$. Most of Lockeia species previously reported are small in size; $10 \mathrm{~mm}$ (maximum size of holotype of $L$. amygdaloides (Seilacher, 1953), $13 \mathrm{~mm}$ (maximum of paratype materials of $L$. siliquaria (Osgood, 1970), $13 \mathrm{~mm}$ (El-Khayal and Romano, 1988), $14 \mathrm{~mm}$ (Kim, 1994), $15 \mathrm{~mm}$ (Crimes et al., 1981), $20 \mathrm{~mm}$ (Chamberlain, 1971), and $27 \mathrm{~mm}$ (Pickerill, 1977). Lockeia gigantus is clearly distinguished from other ichnospecies of Lockeia by its large dimension, about three times the largest Lockeia ever described, though only size difference is regarded to be insufficient for erection of a new ichnospecies.

The general outline of Lockeia gigantus with seedlike to almond-shaped forms tapering at both ends resemble that of Lockeia siliquaria and $L$. amygdaloides except the size difference. However, Lockeia gigantus is also characterized by prominent longitudinal furrow or groove, $\mathrm{V}$ - or U-shaped form in the transverse section, and marginal rims laterally developed at both sides (Fig. 6).

\section{Discussion}

Except very rare cases where trace fossils occurred with trace maker, it is very hard to interpret exactly the trace maker only from the trace fossils. Mutual exclusive preservation of trace fossils and trace makers is a very common cases in ichnology.

According to Seilacher (1953), diagnosis of Lockeia (Pelecypodichnus) is: "Ruhespuren in der Form hochkant stehender Mandeln". Osgood (1970) also gave similar interpretation of $L$. siliquaria; vertical orientation of antero-posterior axis of pelecypod.

The surface ornamentation in each form may be related with the original trace maker and later environmental and diagenetic changes. Some forms with nearly concentric fine ribs or growth lines which may represent the surface ornamentation of original shells may partly be related with the slightly upward or downward movement of animals in response to environmental changes. The longitudinal furrows are considered to be structures representing ventral and lateral commissure of shells, like Pelecypodichnus from the Carboniferous strata of England (Eagar et al., 1983).

The longitudinal symmetry of most forms may represent the symmetry between the equivalves of pelecypod. But some specimens show slightly asymmetry in longitudinal section which seems to be related with oblique orientation of shells to sediment surface.

All of the forms are generally biconvex in outline. The wide range of convexity of forms from strongly biconvex to slightly biconvex is noted. The convexity, as well as overall shape, of forms may undoubtedly represent that of shells. But even within the same shells, they seem to be related with the burrowing depth of shell below the sediment surface and degree of sediment erosion. The more biconvex forms are considered to be produced in deeper burrowing of shells or more slight erosion of sediments.

Most forms show tapering at one end or at both ends which is known as one of diagnosis of Lockeia (James, 1879). These tapering ends are supposed to represent the ventral or lateral commissure of shells. The tapering ends of a few specimens are curved in shape which seems to be related to slight change of shell position. Thus, the tapering of one end or both ends is considered to represent the orientation of shells; tapering only at one end may be related with oblique orientation, i.e. anterior obliquely down and posterior obliquely up, of shells, whereas tapering at both ends in the specimens from the Jinju Formation may be related with nearly horizontal orientation, i.e. ventral down and dorsal up, of shells.

Hardy (1970), Eagar (1971, 1974), Hudson (1980), Eagar et al. (1983, 1985), Thoms and Berg (1985), and Wright and Benton (1987) recognized the bivalves Carbonicola, Neomiodon, Archanodon, and Eotrapezium as likely producers of some forms of Lockeia from the Carboniferous of England, the Jurassic of Scotland, the Devonian of the eastern USA, and the Triassic of England, respectively.

Over 450 specimens of Lockeia are occasionally associated with a bivalve Plicatounio, the most-likely 
producers of them (Fig. 6F). Plicatounio is one of the most common bivalves frequently reported from the Early Cretaceous Jinju Formation in the Gyeongsang Basin which has well known as one of the most significant dinosaur track sites in the world (e.g. Yang, 1979; Lockley et al., 2006). Plicatounio is a medium sized (about $50 \mathrm{~mm}$ in length), elongated elliptical to depressed triangular non-siphonate non-marine bivalve lived on muddy sand in the shallow water. It is characterized by the distinct radial ribs on the surface and the marginal crenation on the inner side of the shell (Suzuk, 1943). Therefore, Lockeia gigantus provides some clue for interpretation of fossil behavior (making communities, resting and shallow burrowing) of Plicatounio as the most-likely producer of $L$. gigantus.

\section{Acknowledgment}

This study was financially supported by the Cultural Heritage Administration of Korea as "Research on the fossils in the southern coast of Korea". Authors thank Dr. C.Z. Lee and an anonymous reviewer for useful comments on the original draft of this paper. Authors also thank Miss S. H. Kim, S. J. Lee, H. J. Park, H. S. Kim, and J. O. Woo for helping in the field and laboratory,

\section{References}

Archer, A.W. and Maples, C.G, 1984, Trace fossil distribution across a marine to nonmarine gradient in the Pennsylvanian of southwestem Indiana. Journal of Paleontology, 5, 448-466.

Bandel, K., 1967, Trace fossils from two upper Pennsylvanian sandstones in Kansas. University of Kansas Paleontological Contribution, 18, 1-13.

Bjerstedt, T.W., 1987, Latest Devonian-Earliest Mississippian near-shore trace assemblages from West Virginia, Pennsylvania and Maryland. Journal of Paleontology, $61,865-889$.

Bjerstedt, T.W., 1988, Trace fossils from the early Mississippian Price Delta, Southeast West Virginia. Journal of Paleontology, 62, 506-519.

Bromley, R. and Asgaard, U., 1979, Triassic fresh water ichnocoenoses from Carlsberg Fjord, East Greenland.
Palaeogeography, Palaeoclimatology, Palaeoecology, 28, 39-80.

Chamberlain, C.K., 1971, Morphology and ethology of trace fossils from the Ouachita Mountains, southeast Oklahoma. Journal of Paleontology, 45, 212-246.

Chamberlain, C.K., 1978, Trace fossil ichnofacies of an American flysch. In Chamberlain, C.K. (ed.), A guidebook to the trace fossils and paleoecology of the Ouachita Geosyncline. Society of Economic Paleontologists and Mineralogists, 23-37.

Chang, K.H., 1975, Cretaceous stratigraphy of southeast Korea. Journal of the Geological Society of Korea, 11, $1-23$.

Chang, K.H., 1977, Late Mesozoic stratigraphy, sedimentation and tectonics of southern Korea. Journal of the Geological Society of Korea, 13, 76-90.

Chang, K.H., 1987, Cretaceous strata. In Lee, D.S. (ed.), Geology of Korea. Geological Society of Korea, Seoul, 157-210.

Chang, K.H., 1988, Cretaceous stratigraphy and paleocurrents analysis of Kyongsang Basin, Korea. Journal of the Geological Society of Korea, 24, 194-205.

Chang, K.H. and Kim, H.M., 1968, Cretaceous paleocurrents and sedimentation in northwestern part of Gyeongsang Basin, southern Korea. Journal of the Geological Society of Korea, 4, 77-97.

Chang, K.H., Woo, B.G., Lee, J.H., Park, S.O., and Yao, A., 1990. Cretaceous and early Cenozoic stratigraphy and history of eastern Kyongsang Basin, Southern Korea. Journal of the Geological Society of Korea, 26, 471-487.

Chaplin, J.R., 1980, Stratigraphy, trace fossil associations, and depositional environments in the Borden Formation (Mississippian), northeastern Kentucky. Annual Field Conference of the Geological Society Kentucky, 1980, Kentucky Geological Survey, 114 p.

Chaplin, J.R., 1982, Paleoenvironments and biostratigraphy of the Borden and parts of the Newman and Breathitt formations (Mississippian-Pennsylvanian) in northeastern Kentucky. Tewltth Annual Field Conference, Great Lakes Section, Society of Economic and Paleontologists and Mineralogists, $196 \mathrm{p}$.

Cheong, D.K. and Kim, Y.I., 1996, Sedimentological study of the Nakdong Formation to analyse the forming and evolving tectonics of the Cretaceous Gyeongsang Basin. I. Depositional setting, source, and paleocurrent analyses of the Nakdong Formation in the southwestern Gyeongsang Basin. Economic Environmental Geology, 29, 639-660.

Choi, D.K., 1985, Spores and pollen from the Gyeongsang Supergroup, southeastern Korea and their chronologic and paleoecologic implications. Journal of the Paleontological Society of Korea, 1, 33-50. 
Choi, D.K., 1989, Paleopalynology of the Geoncheonri Formation (Lower Cretaceous), Geoncheon-Ahwa area, Korea. Journal of Paleontological Society Korea, 5, 127.

Choi, H.I., 1986a, Sedimentation and evolution of the Cretaceous Gyeongsang Basin, southeastern Korea. Journal of the Geological Society, 143, 29-40.

Choi, H.I., 1986b, Sandstone petrology of the Sindong Group, southwestern part of the Gyeongsang Basin. Journal of the Geological Society of Korea, 22, 212223.

Choi, H.I., 1986c, Fluvial plain/lacustrine facies transition in the Cretaceous Sindong Group, south coast of Korea. Sedimentary Geology, 48, 295-320.

Choi, P.Y., Choi, H.I., Hwang, J.H., Kee, W.S., Koh, H.J., Kim, Y.B., Lee, B.J., Song, K.Y., Kim, J.C., and Choi, Y.S., 2002, 1:250,000 Explanatory note of the Mokpo and Yeosu sheets. Korea Institute of Geoscience and Mineral Resources, $44 \mathrm{p}$.

Choi, S.J., 1989, Fossil charophytes from the Nagdong Formation in Seonsangun, Gyeongsangbukdo, Korea. Journal of Paleontological Society Korea, 5, 28-38.

Choi, S.J., 1990, Some nonmarine fossils from the central part of Gyeongsang Basin, S. Korea. Journal of the Paleontological Society of Korea, 6, 165-179.

Costa, G.D. and Muniz, B., 1979, Novos ichnofosseis Devonianos da Formacao Inaja, no Estado de Pernambuco. Anals de Academie brasil Cienc, 51, 121-132.

Crimes, T.P., 1977, Trace fossils of an Eocene deep-sea sand fan, northern Spain. In Crimes, T.P. and Harper, J.C. (eds.), Trace Fossils 2. Geological Journal Special Issue, 9, 71-90.

Crimes, T.P., Goldring, R., Homewood, P., Van Stuijvenberg, J., and Winkler, W., 1981, Trace fossil assemblages of deep-sea fan deposits, Gurnigel and Schlieren flysch (Cretaceous-Eocene), Switzerland. Ecologae Geologicae Helvetiae, 74, 953-995.

Doh, S.J. and Kim, K.H., 1994, A paleomagnetic study of Cretaceous rocks from the Euiseong area. Economic and Environmental Geology, 27, 263-279.

Eagar, R.M.C., 1971, A new section in the Lower Coal Measures (Westpalian A) of Up Holland, near Wigan, Lancashire. Proceedings of Geologists Association, 82, 71-85.

Eagar, R.M.C., 1974, Shape of shell of Carbonicola in relation to burrowing. Lethaia, 7, 219-238.

Eagar, R.M.C., Okolo, S.A., and Walters, G.E., 1983, Trace fossils as evidence in the evolution of Carbonicola. Proceedings of the Yorkshire Geological Society, 44, 283-303.

Eagar, R.M.C., Baines, J.C., Collinson, J.D., Hardy, P.G., Okolo, S.A., and Pollard, J.E., 1985, Trace fossil assemblages and their occurrence in Silesian (Mid-Car- boniferous) deltaic sediments of the central Pennine Basin, England. In Curran, H.A. (ed.), Biogenic structures: Their use in interpreting depositional environments. Society of Economic Paleontologists Special Publication, 35, 99-149.

El-Khayal, A.A. and Romano, M., 1988, A revision of the upper part of the Saq Formation and Hanadir Shale (Lower Ordovician) of Saudi Arabia. Geological Magazine, 125, 161-174.

Fillion, D. and Pickerill, R.K., 1990, Ichnology of the Upper Cambrian? to Lower Ordovician Bell Island and Wabana groups of eastern Newfoundland, Canada. Palaeontographica Canadiana, 7, $119 \mathrm{p}$.

Gong, Y. and Liu, B., 1993, Plate-tectonic sedimentology of the Devonian volcanic sedimentary successions in northern Xinjiang, northwestern China. The Press of the China University of Geosciences, $138 \mathrm{p}$.

Hakes, W.G., 1976, Trace fossils and depositional environments of four clastic units, Upper Pennsylvanian megacyclothems, northeast Kansas. University of Kansas Paleontological Contributions, 63, 1-46.

Hakes, W.G., 1985, Trace fossils from brackish-marine shales, Upper Pennsylvanian of Kansas, U.S.A. In Curran, H.A. (ed.), Biogenic structures: Their use in interpreting depositional environments. Society of Economic Paleontologists Special Publication, 35, 21-35.

Häntzschel, W., 1975, Trace fossils and problematica. In Teichert, C. (ed.), Treatise on invertebrate paleontology, Part W, Miscellanea, Supplement 1. Geological Society of America and University of Kansas Press, 269 p.

Hardy, P.G, 1970, New xiphosurid trails from the Upper Carboniferous of northern England. Palaeontology, 13, 188-190.

Hudson, J.D., 1980, Aspects of brackish-water facies and faunas from the Jurassic of north-west Scotland. Proceedings of the Geologists Association, 91, 99-105.

James, U.P., 1879, Description of new species of fossils and remarks on some others, from the Lower and Upper Silurian rocks of Ohio. The Paleontologist, 3, 17-24.

Kamola, D.L., 1984, Trace fossils from marginal marine facies of the Spring Canyon Member, Blackhawk Formation (Upper Cretaceous, east-central Utah). Journal of Paleontology, 58, 529-541.

Karaszewski, W., 1974, A new trace fossil from the Lower Jurassic of the Holy Cross Mountains. Acadmie Polonaise des Sciences, Bulletin, Srie des Sciences de la Terre, 22, 157-160.

Kim, D.H., Hwang, J.H., Park, K.H., and Song, K.Y., 1998, 1:250,000 Explanatory note of the Pusan sheet. Ministry of Science and Technology, $62 \mathrm{p}$.

Kim, J.Y., 1994, A unique occurrence of Lockeia from the Yeongheung Formation (Middle Ordovician), Yeong- 
weol, Korea. Ichnos, 3, 219-225.

Lockley, M.G., Houck, K., Yang, S.Y., Matsukawa, M., and Lim, S.K., 2006, Dinosaur-dominated footprint assemblages from the Cretaceous Jindong Formation, Hallyo Haesang National Park area, Goseong County, South Korea: Evidence and implications. Cretaceous Research, 27, 70-101.

MacEachern, J.A., Zaitlin, B.A., and Pemberton, S.G., 1994, Ichnological aspects of incised-valley fill systems from the Viking Formation of the Western Canada sedimentary basin, Alberta, Canada. Society of Economic Paleontologists and Mineralogists, Special Publication, 51, 129-157.

MacEachern, J.A., Zaitlin, B.A., and Pemberton, S.G., 1998, High-resolution sequence stratigraphy of early transgressive deposits, Viking Formation, Joffre Field, Alberta, Canada. The American Association of Petroleum Geologists, 82, 729-756.

MacEachern, J.A., Zaitlin, B.A., and Pemberton, S.G., 1999a, A sharp-based sandstone of the Viking Formation, Joffre Field, Alberta, Canada: Criteria for recognition of transgressively incised shoreface complexes. Journal of Sedimentary Research, 69, 876-892.

MacEachern, J.A., Zaitlin, B.A., and Pemberton, S.G., 1999b, Marine and marginal marine mudstone deposition: Paleoenvironmental interpretations based on the integration of ichnology, palynology and foraminiferal paleoecology. Society of Economic Paleontologists and Mineralogists, Special Publication, 64, 205-225.

MacEachern, J.A., Zaitlin, B.A., and Pemberton, S.G., 1999c, Coarse-grained, shoreline-attached, marginal marine parasequences of the Viking Formation, Joffrefield, Alberta, Canada. Society of Economic Paleontologists and Mineralogists, Special Publication, 64, 273296.

Maples, C.G. and Suttner, L.J., 1990, Trace fossils and marine-nonmarine cyclicity in the Fountain Formation (Pennsylvanian: Morrowan/Atokan) near Manitou Spring, Colorado. Journal of Paleontology, 64, 859-880.

Miller, M.F., 1984, Distribution of biogenic structures in Paleozoic nonmarine and marine-margin sequences: An actualistic model. Journal of Paleontology, 58, 550-570.

Narbonne, GM. and Aitken, J.D., 1990, Ediacaran fossils from the Sekwi Brook area, Mackenzie Mountains, northwestern Canada. Palaeontology, 33, 945-980.

Osgood, R.G, 1970, Trace fossils of the Cincinnati area. Palaeontographica Americana, 6, 281-444.

Paik, I.S. and Chun, J.H., 1993, Laminar calcretes, calcrete pisoids and ooids, and rhizoliths from the Kyeongsang Supergroup, Korea. Journal of the Geological Society of Korea, 29, 108-117.

Paik, I.S. and Lee, Y.I., 1994, Paleoclimatic records in floodplain lake deposits of the Cretaceous Hasandong
Formation in Jinju area, Korea. Journal of the Geological Society of Korea, 30, 410-424.

Paik, I.S. and Kim, J.Y., 1995, Calcareous paleosols from the Upper Hasandong Formation in the vicinity of Jinju, Korea: Implications in floodplain sedimentation and paleoclimate. Journal of the Geological Society of Korea, 31, 482-498.

Paik, I.S. and Lee, Y.I., 1998, Desiccation cracks in vertic paleosols of the Cretaceous Hasandong Formation, Korea: Genesis and paleoenvironmental implications. Sedimentary Geology, 119, 161-179.

Pickerill, R.K., 1977, Trace fossils from the Upper Ordovician (Caradoc) of the Berwyn Hills, Central Wales. Geological Journal, 12, 1-16.

Pienkowski, G., 1985, Early Liassic trace fossil assemblages from the Holy Cross Mountains, Poland: Their distribution in continental and marginal marine environments. In Curran, H.A. (ed.), Biogenic structures: Their use in interpreting depositional environments. Society of Economic Paleontologists and Mineralogists, Special Publication, 35, 37-51.

Pienkowski, G., 1991, Liassic sedimentation in Scania, southern Sweden: Hettangian-Sinemurian of the Helsingborg area. Facies, 24, 40-85.

Pryor, W.A., 1967, Biogenic directional features on several Recent pointbars. Sedimentary Geology, 1, 235-248.

Rindsberg, A.K., 1994, Ichnology of the Upper Mississippian Hartselle Sandstone of Alabama, with notes on other Carboniferous formations. Geological Survey of Alabama Bulletin, 158, 1-107.

Seilacher, A., 1953, Studien zur Palichnologie II. Die fossilen Ruhespuren (Cubichnia). Neues Jahrbuch für Geologie und Palontologie Abhandlungen, 98, 87-124.

Suzuki, K., 1943, Restudy on the non-marine molluscan fauna of the Rakuto Series in Keisyo-do, Tyosen. Journal of the Sigenkagaku Kenkyusyo, 1, 189-219.

Tateiwa, I., 1925, Geological age of the Nagdong flora. Journal of the Geological Society of Tokyo, 32, 493512.

Tateiwa, I., 1929, Geological atlas of Korea, no. 10, Kyongju, Yongchon, Taegu, and Waegwan sheets. Geological Survey of Chosen, $12 \mathrm{p}$.

Thoms, R,E. and Berg, T.M., 1985, Interpretation of bivalve trace fossils in fluvial beds of the basal Catskill Formation (late Devonian), eastern U.S.A. In Curran, H.A. (ed.), Biogenic structures: Their use in interpreting depositional environments. Society of Economic Paleontologists and Mineralogists, Special Publication, 35, 13-20.

Vossler, S.M. and Pemberton, S.G., 1988, Ichnology of the Cardium Formation (Pembina Oilfield): Implications for depositional and sequence stratigraphic interpretations. In James, N.P. and Leckie, D.A. (eds.), Sequences, 
stratigraphy, sedimentology: Surface and sub-surface. Canadian Society of Petroleum Geologists Memoir, 15, 237-254.

Wincierz, J., 1973, Kustensedimente und Ichnofauna aus dem oberem Hettangium von Mackendorf (Niedersachsen). Neues Jahrbuch für Geologie und Palontologie Abhandlungen, 98, 104-141.

Wright, A.D. and Benton, M.J., 1987, Trace fossils from Rhaetic shoreface deposits of Staffordshire. Palaeontology, 30, 407-428.

Yang, S., 1984, Silurian trace fossils from the Yangzi Gorges and their significance to depositional environments. Acta Palaeontologica Sinica, 23, 705-714.

Yang, S.Y., 1974, Note on the genus Trigonioides (Bivalvia). Transactions and Proceedings of the Paleontological Society of Japan, 95, 395-408.

Yang, S.Y., 1975, On a new non-marine pelecypod genus from the Upper Mesozoic Gyeongsang Group of Korea. Transactions and Proceedings of the Paleontological Society of Japan, 100, 177-184.

Yang, S.Y., 1976, On fossils from the Gyeongsang Groupespecially on some of the molluscan fauna. Journal of the Geological Society of Korea, 12, 23-30.
Yang, S.Y., 1978a, Ontogenetic variation of Trigonioides (s.s.) paucisulactus (Cretaceous non-marine Bivalvia). Transactions and Proceedings of the Paleontological Society of Japan, 111, 333-347.

Yang, S.Y., 1978b, On the discovery of Nippononaia ryosekiana from the Gyeongsang Group, Korea. Journal of the Geological Society of Korea, 14, 33-43.

Yang, S.Y., 1979, Some new bivalve species from the Lower Gyeongsang Group. Transactions and Proceedings of the Paleontological Society of Japan, 116, 223234.

Yang, S.Y., 1982, Geology around the type-locality of Trigonioides. Journal of the Geological Society of Korea, $18,67-72$.

Yi, M.S., Choi, S.J., and Yun, H., 1993, Cretaceous palynomorphs from the Iljik Formation in the Euiseong area, Korea. Journal of the Paleontological Society of Korea, 9, 166-179.

Yi, M.S., Cho, B.H., and Chi, J.M., 1994, Palynomorphs from the Jinju Formation in the Euiseong area, Korea. Journal of the Paleontological Society of Korea, 10, 4156. 